\title{
Understanding patient perspectives of the impact of anaemia in chronic kidney disease: A United States patient survey
}

\author{
Susan Grandy $^{1}$, Eirini Palaka ${ }^{2}$, Nicolas Guzman ${ }^{3}$, Alicia Dunn ${ }^{3}$, Eric Wittbrodt ${ }^{4}$, and \\ Fredric Finkelstein ${ }^{5}$
}

${ }^{1}$ AstraZeneca Pharmaceuticals LP

${ }^{2}$ AstraZeneca Ltd

${ }^{3}$ Astrazeneca

${ }^{4}$ AstraZeneca

${ }^{5}$ Yale University

October 8, 2020

\begin{abstract}
Background Anaemia, a common complication of chronic kidney disease (CKD), is associated with reduced health-related quality of life and physical functioning. This study investigated the knowledge, perceptions and awareness of anaemia in patients with CKD in the United States. Methods Between 29 August and 17 September 2018, an online survey was administered to consenting patients in the United States aged [?] 18 years with self-reported CKD, with or without anaemia; patients with cancer were excluded. The survey comprised 27 questions, exploring anaemia knowledge, health-related quality of life, anaemia management, practitioner-patient relationships, and the information sources patients used. Results Of 446 patients with CKD, 255 (57.2\%) were diagnosed with anaemia and 191 (42.8\%) reported not being diagnosed with anaemia, or were unsure/could not remember (non-anaemia cohort). In patients with anaemia, $71.0 \%$ were aware of the relationship between CKD and anaemia versus $52.9 \%$ of those in the non-anaemia cohort. In the anaemia cohort, $46.3 \%$ of patients were aware of their haemoglobin level, compared with $27.2 \%$ of patients in the non-anaemia cohort. Symptoms most commonly reported as being made worse by anaemia included lack of energy $(82.4 \%)$, feeling ill (31.4\%), and the ability to enjoy life (30.2\%). Despite $67.4 \%$ of patients with anaemia in CKD believing that their condition was well or very well managed, only $50 \%$ reported being informed about different treatments without having to prompt their healthcare professional, and $43.9 \%$ were seeking more information and support about managing their condition. Conclusions In the United States, patients with anaemia and CKD perceived that anaemia had a negative impact on their physical health and emotional wellbeing. Our findings emphasize the lack of disease awareness regarding anaemia in patients with CKD, suggesting that patients would benefit from further education regarding its management, and available treatment options.

Susan Grandy, ${ }^{1}$ Eirini Palaka, ${ }^{2}$ Nicolas Guzman, ${ }^{3}$ Alicia Dunn, ${ }^{4}$ Eric T. Wittbrodt, ${ }^{5}$ Fredric O. Finkelstein ${ }^{6}$

${ }^{1}$ Biopharmaceuticals Global Market Access, AstraZeneca, Gaithersburg, MD, USA ${ }^{2}$ Biopharmaceuticals Global Market Access, AstraZeneca, Cambridge, UK ${ }^{3}$ Global Medicines Development, AstraZeneca, Gaithersburg, MD, USA ${ }^{4}$ Global Corporate Affairs, AstraZeneca, Gaithersburg, MD, USA ${ }^{5}$ Biopharmaceuticals Medical Unit, AstraZeneca, Gaithersburg, MD, USA ${ }^{6}$ Yale University, New Haven, CT, USA
\end{abstract}

Running title : US patient perspectives of anemia in CKD

Correspondence : Dr Susan Grandy, BioPharmaceuticals Business Unit, AstraZeneca, One MedImmune Way, Gaithersburg, MD 20878. Tel: +1 302 723-1523; Email: susan.grandy@astrazeneca.com

Abstract word count: 293 
Word count: $2865(5000 \max )$

\section{Acknowledgments}

We would like to thank the patients who participated in this study. Medical writing support was provided by Mahalia Gilmartin, PhD and India Wright, MSc, and editorial support was provided by Rachael Cazaly, BSc, all of Core Medica, London, UK, supported by AstraZeneca according to Good Publication Practice guidelines (link). The sponsor was involved in the study design and collection, analysis and interpretation of data, as well as data checking of information provided in the manuscript. However, ultimate responsibility for opinions, conclusions and data interpretation lies with the authors. Development of this manuscript was supported by AstraZeneca.

\section{Disclosures}

E. Palaka, N. Guzman, A. Dunn, E.T. Wittbrodt and S. Grandy are employees and stockholders of AstraZeneca. F.O. Finkelstein has received consultation fees from AstraZeneca, GSK,and Akebia.

\section{Author contributions}

A. Dunn, S. Grandy, E. Palaka and E.T. Wittbrodt contributed to the study design and data collection. E.T. Wittbrodt, E. Palaka, F.O. Finkelstein, N. Guzman and S. Grandy performed the data analysis and interpretation. All authors critically reviewed the manuscript, approved the final version, and accept accountability for the overall work.

\section{Data sharing statement}

Data underlying the findings described in this manuscript may be obtained in accordance with AstraZeneca's data sharing policy described at: https://astrazenecagrouptrials.pharmacm.com/ST/Submission/Disclosure.

\section{Hosted file}

Grandy_US patient survey manuscript_main text28Sept20_IW.pdf available at https://authorea. com/users/365505/articles/485580-understanding-patient-perspectives-of-the-impact-ofanaemia-in-chronic-kidney-disease-a-united-states-patient-survey 
Figure 1. Beliefs about (A) causes of anaemia, and (B) symptoms associated with anaemia

A

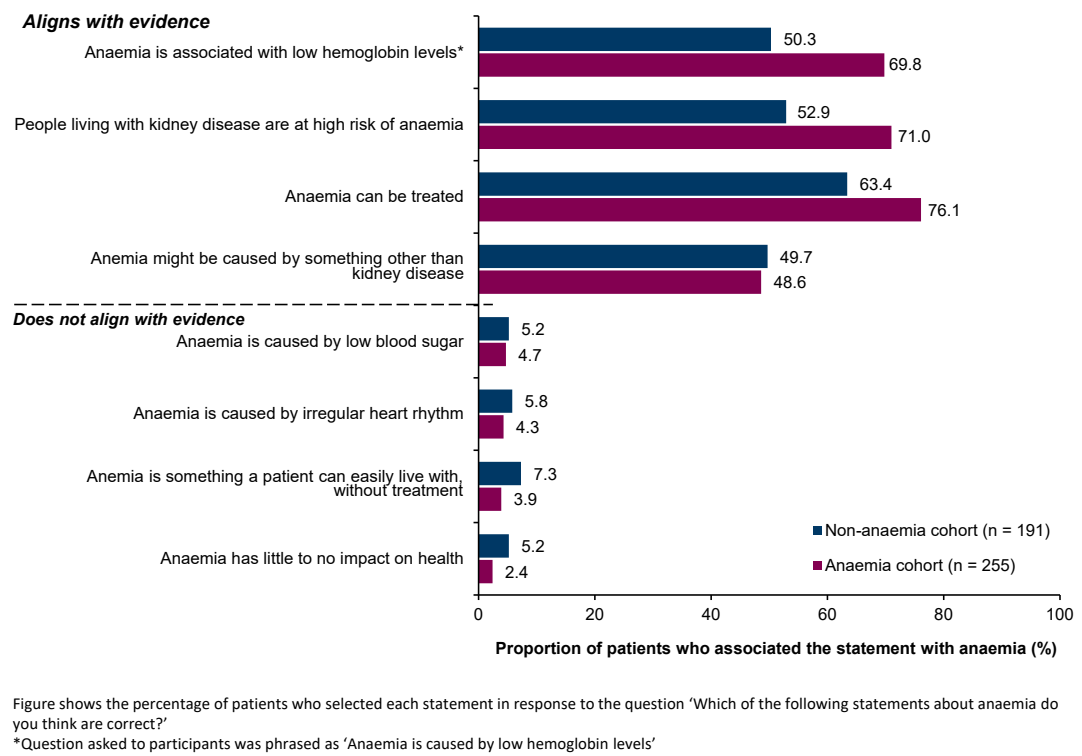

B

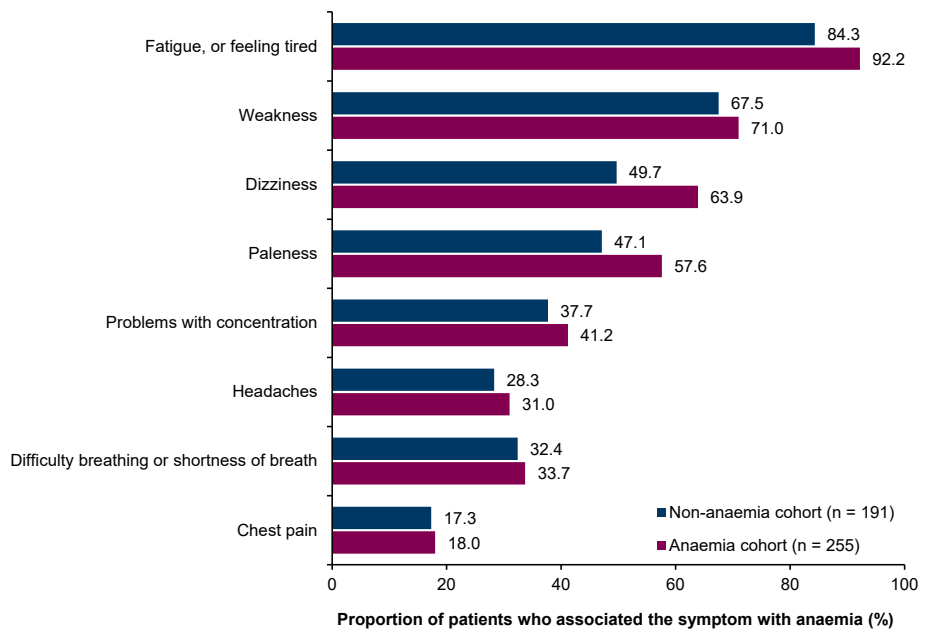

Figure shows the percentage of patients who selected each statement in response to the question 'Which of the following do you think are symptoms of anaemia? 
Figure 2. (A) Perception of chronic disease impact on HRQoL (anaemia cohort, $n=255)$ : $(B)$ worsening of physical symptoms attributed to anaemia by these patients

A

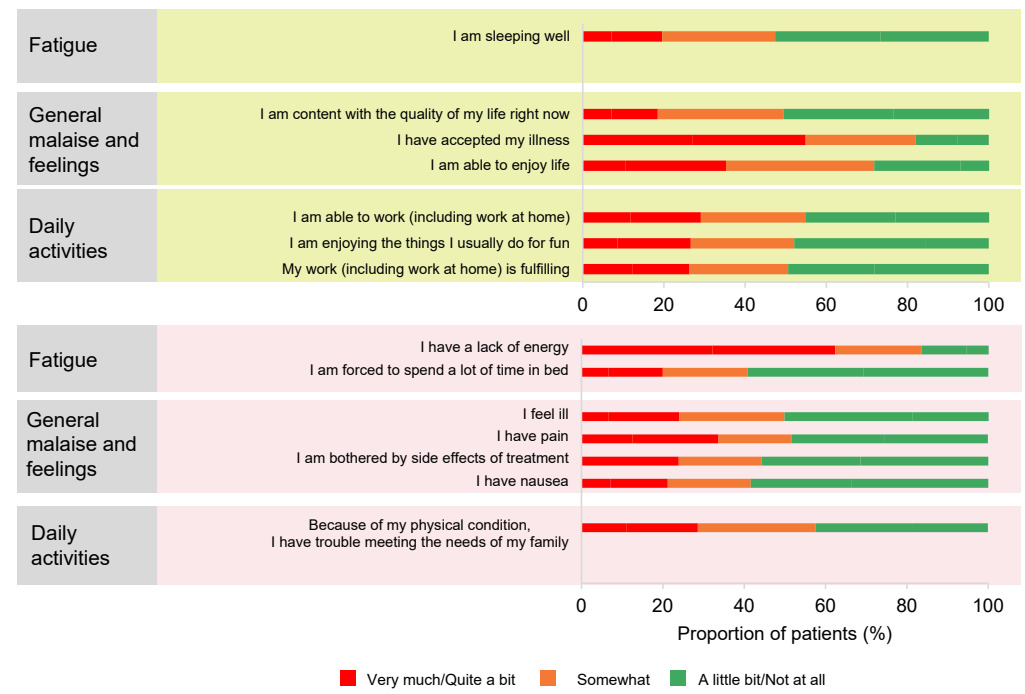

Patients were asked the following question 'Below are feelings that people who have a chronic condition, such as kidney disease, have mentioned that they can relate to. Please tell us how much these apply to you on a scale of 1 to 5 , with 1 being 'not at all' and 5 'very
much".

B

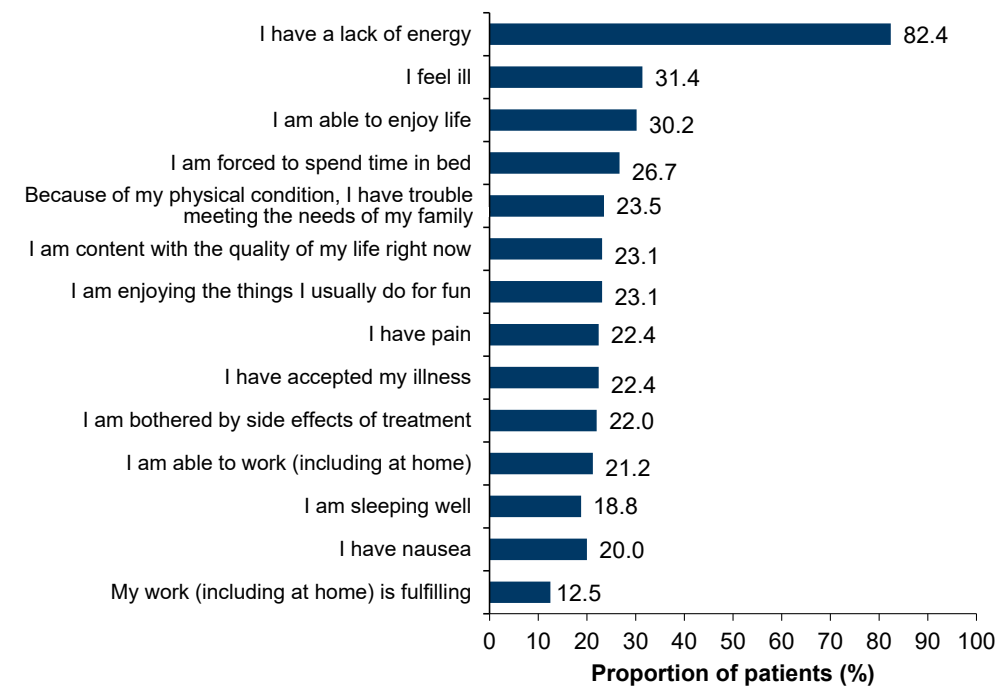

Abbreviation: HRQoL, health-related quality of life.

Patients were asked the following question 'Which of these feelings would you say is made worse by your anaemia?' 
Figure 3. (A) Perception of chronic disease impact on HRQoL (anaemia cohort, $n=255$ ): (B) worsening of emotional symptoms attributed to anaemia in CKD by these patients

A

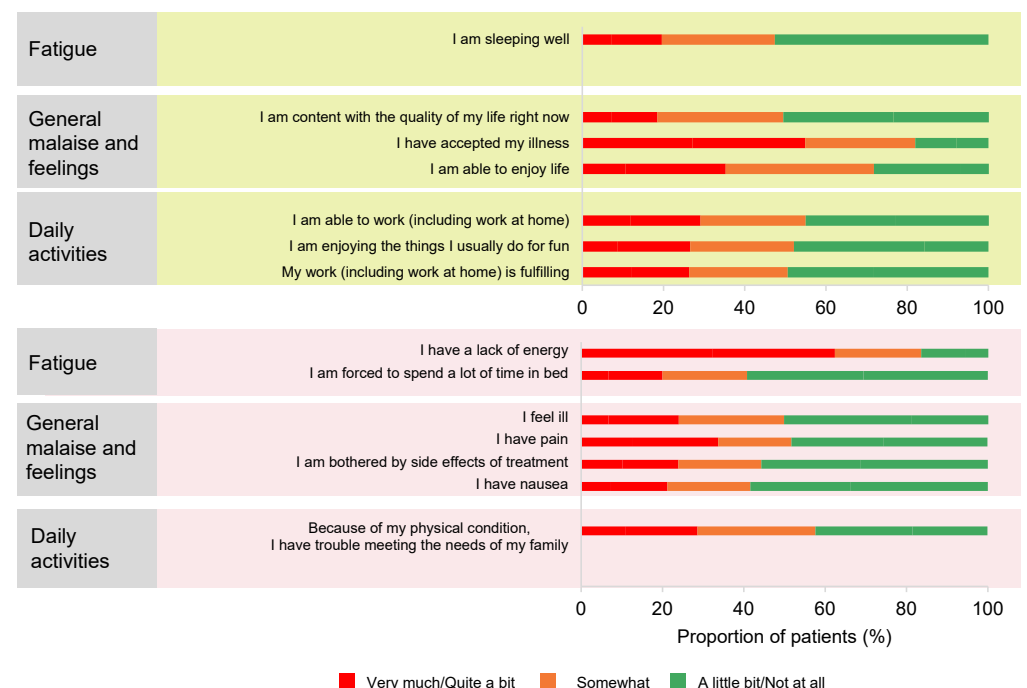

Very much/Quite a bit $\quad$ Somewhat A little bit/Not at all

Abbreviation: $H R Q \circ L$, health-related quality of life.

Patients were asked the following question 'Below are feelings that people who have a chronic condition, such as kidney disease, have mentioned in relation to their emotions. Please tell us how much these apply to you on a scale of 1 to 5 , with 1 being 'not at all' and 5 'very much".

B

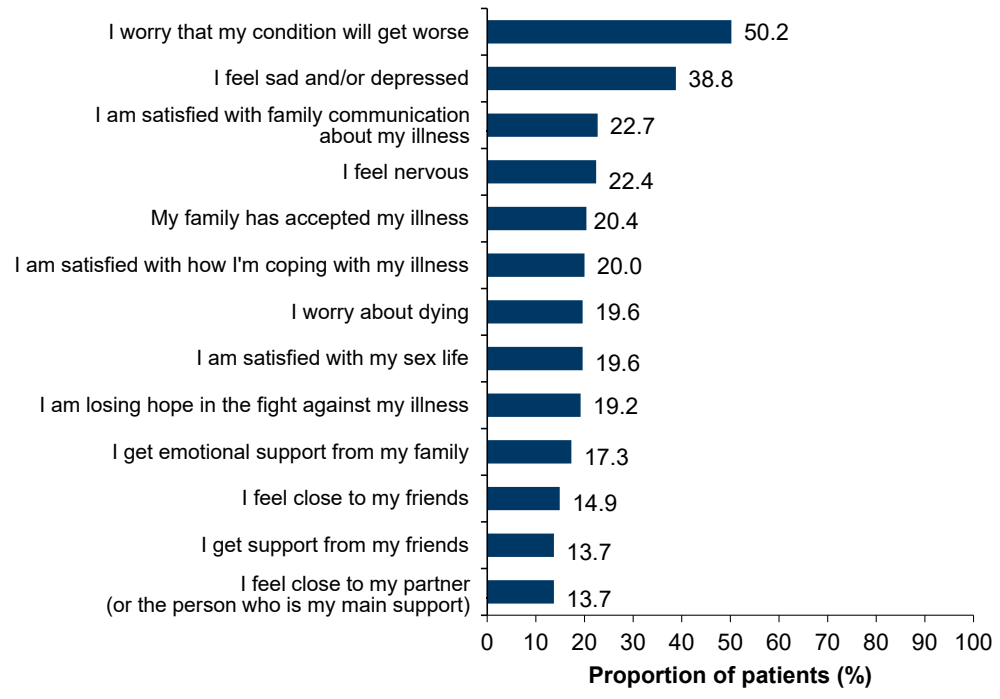

Patients were asked the following question 'Which of these feelings would you say is made worse by your anaemia?' 
Figure 4. (A) Knowledge of treatment options for anaemia and how this was acquired, and $(B)$ anaemia treatments reported by patients

A

Anaemia cohort $(n=255)$

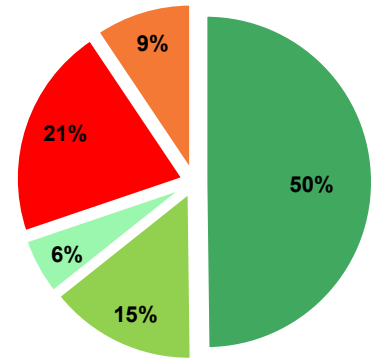

Non-anaemia cohort $(n=191)$

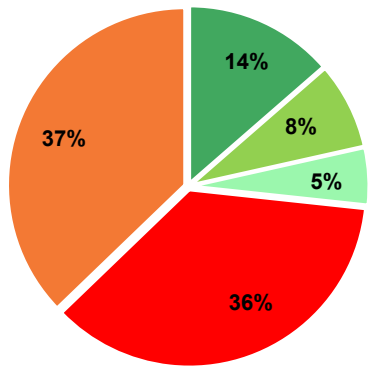

Yes, my doctor informed me about different treatment options for anaemia

Yes, I asked the doctor about treatment options for anaemia

Yes, I found out myself about anaemia treatments

No, but I don't feel I need to know about anaemia treatments

No, but I would like to know

Patients were asked the following question 'Do you know about the different treatments which are available for managing anaemia and, if so, how did you find out?

B

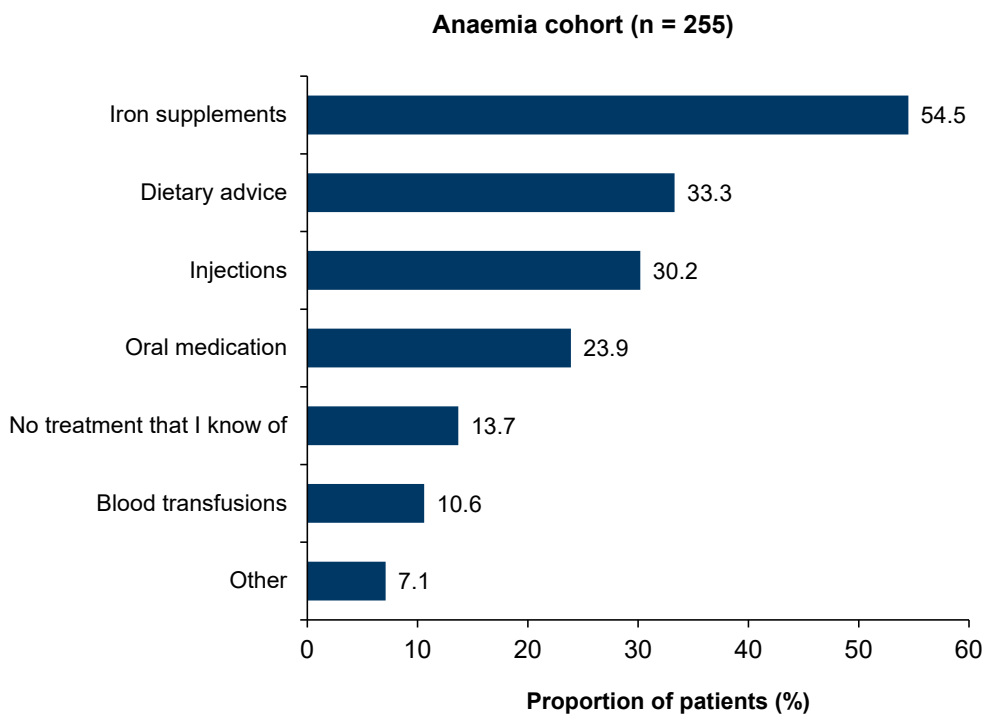

Patients were asked the following question 'How is your doctor currently managing your anaemia?' 
Figure 5. For the anaemia cohort $(n=255)$ : (A) Patient perceptions of how well their anaemia was being managed, and (B) impact of discussing anaemia in CKD with HCPs

A

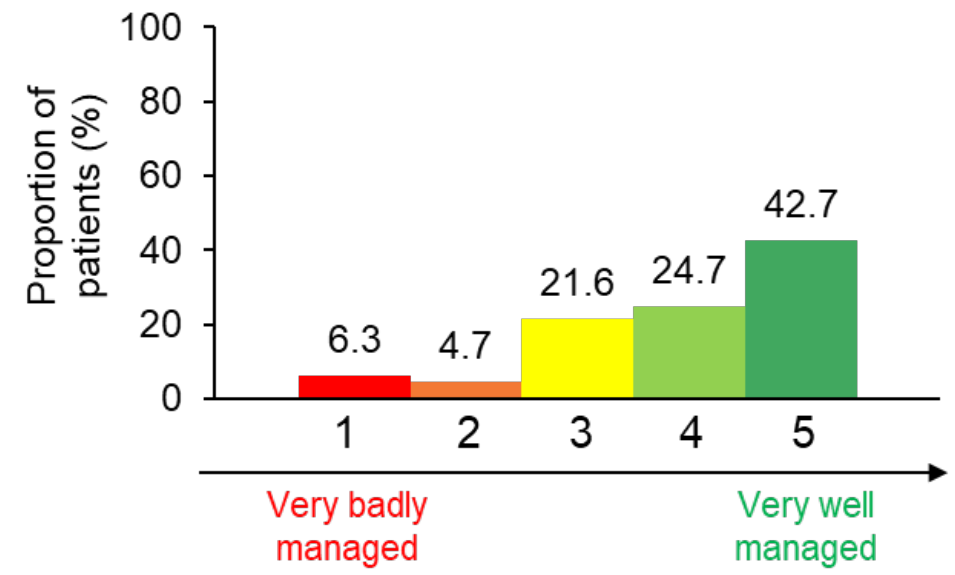

Patients were asked the following question 'On a scale from 1 to 5 , how well do you think your anaemia is managed, with 1 being 'very badly managed', 3 being 'neither badly nor well managed', and 5 being 'very well managed'?'

B

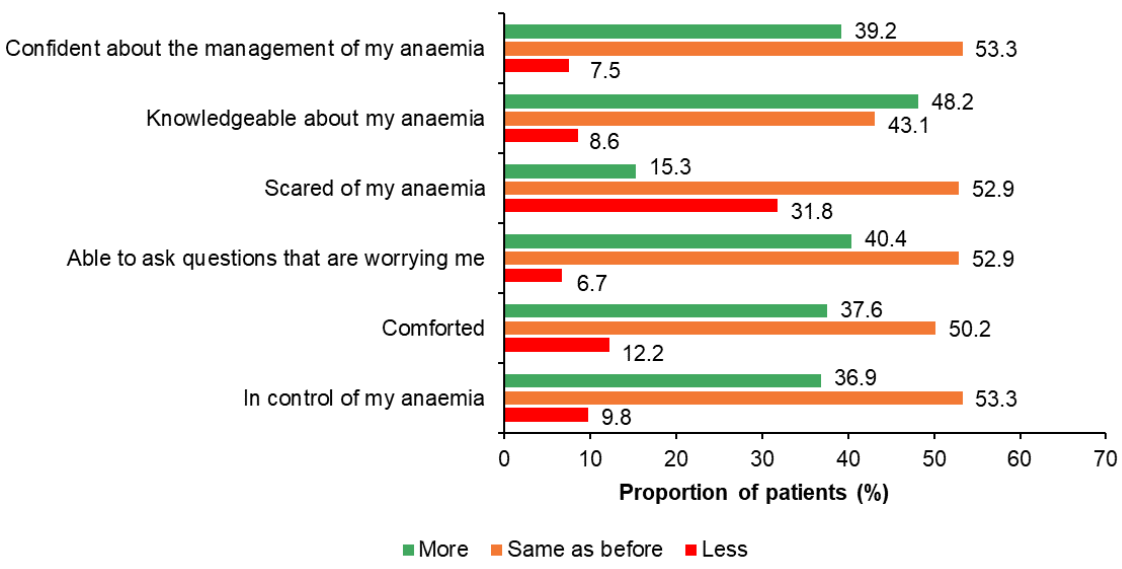

Abbreviations: $\mathrm{CKD}$, chronic kidney disease; $\mathrm{HCP}$, healthcare provider

Patients were asked the following question 'After your discussions with your doctors about anaemia, no matter how small, how do you feel?' 
Figure 6. (A) Sources used by patients to look for information about anaemia, and (B) trust in the information received from these sources

A
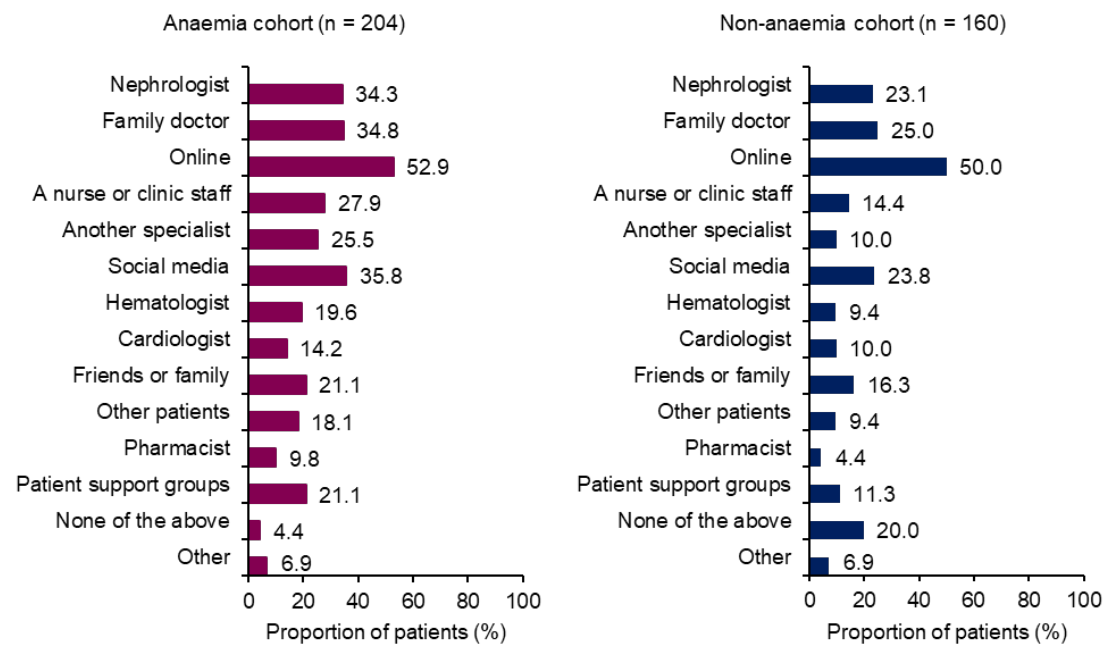

Patients were asked the following question 'Where have you looked for information about anaemia, or received information about anaemia from?'

B
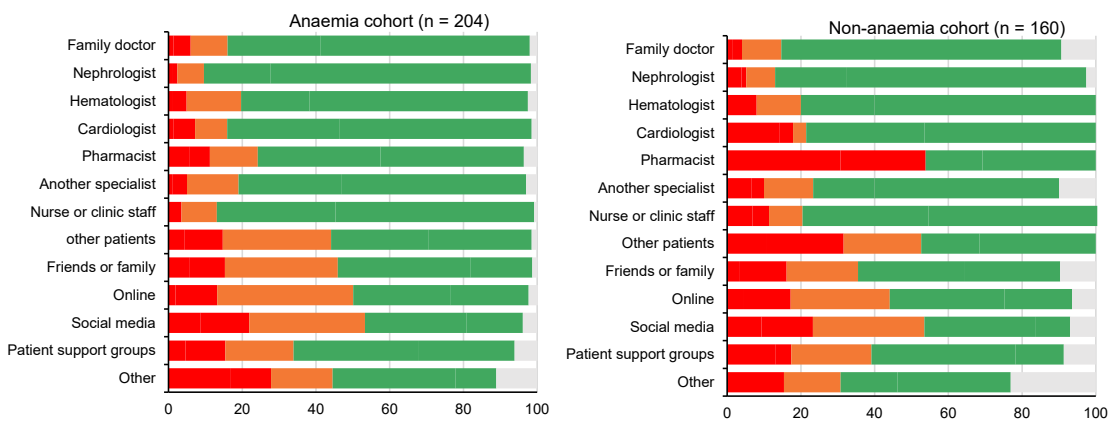

Not at all (1 or 2)

Somewhat (3 or 4$)$

Completely (5) Don't know

Patients were asked the following question 'And how much do you trust the information you get from these sources? From 1 being 'not at all' to 5 being 'completely'?' 
Figure 7. Factors that prevented patients with anaemia in CKD from seeking more information about anaemia (anaemia cohort, $n=255$ )

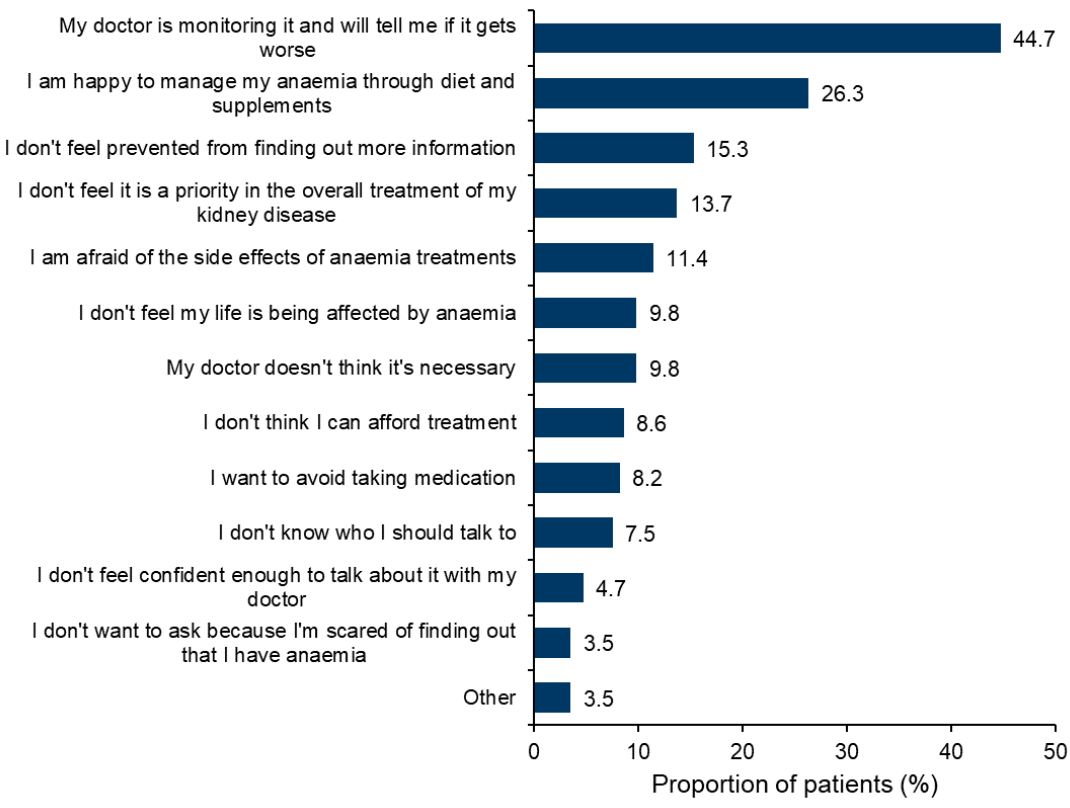

Patients were asked the following question 'What prevents you from seeking help to find out more about your anemia?' 
Figure 8. Factors that would encourage patients to seek more information or greater support about anaemia in CKD (anaemia cohort, $\mathrm{n}=\mathbf{2 5 5}$ )

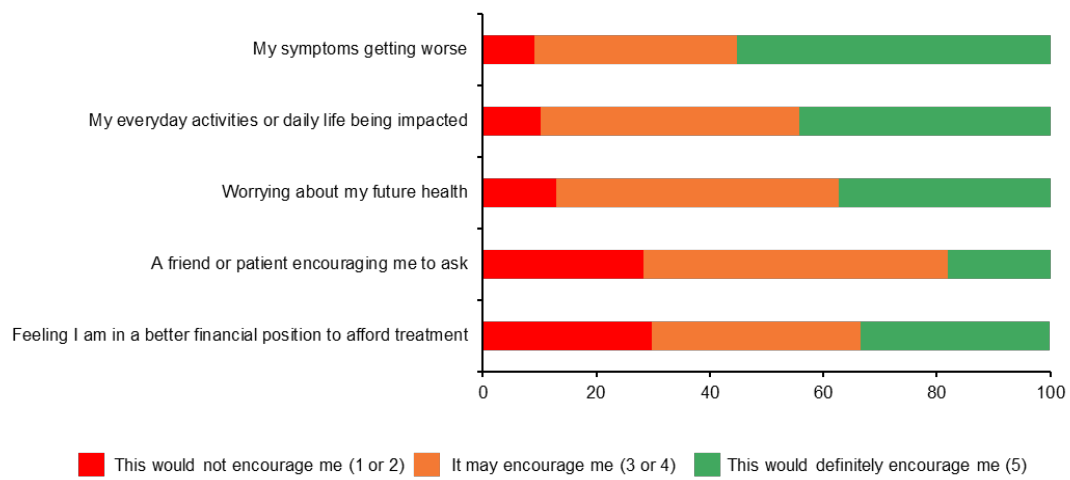

Patients were asked the following question 'What would encourage you to seek more information, or greater support, about anaemia in the context of your kidney disease? Please rate the answers below (rating scale from 1 'this would not encourage me to seek help' to 'this would definitely encourage me to seek help' and middle point 'it may encourage me to seek help')' 